\title{
Tumor suppressor CD99 is downregulated in plasma cell neoplasms lacking CCND1 translocation and distinguishes neoplastic from normal plasma cells and B-cell lymphomas with plasmacytic differentiation from primary plasma cell neoplasms
}

\author{
Qi Gao ${ }^{1} \cdot$ Venkata Yellapantula $^{2} \cdot$ Maly Fenelus $^{3} \cdot$ Janine Pichardo $^{1} \cdot$ Lu Wang $^{4} \cdot$ Ola Landgren $^{5} \cdot$ Ahmet Dogan $^{1}$. \\ Mikhail Roshal ${ }^{1}$
}

Received: 10 June 2017 / Accepted: 6 December 2017 / Published online: 5 February 2018

(c) United States \& Canadian Academy of Pathology 2018

\begin{abstract}
CD99(MIC2) is a widely expressed cell surface glycoprotein and functions as a tumor suppressor involved in downregulation of SRC family of tyrosine kinase. CD99 expression is tightly regulated through B-cell development. The principal aims of this study were to investigate the clinical utility of CD99 expression (i) in distinguishing normal plasma cells from primary plasma cell neoplasms; (ii) in detection of minimal residual disease in primary plasma cell neoplasms; and (iii) in distinguishing plasma cell component of B-cell lymphomas from primary plasma cell neoplasms. We analyzed expression of CD99 by flow cytometry and immunohistochemistry in lymph nodes, peripheral blood, and bone marrow samples. CD99 showed stage-specific expression with highest expression seen in precursor B and plasma cells. In contrast to the uniform bright expression on normal plasma cells, CD99 expression on neoplastic plasma cells was lost in 39 out of 56 (69.6\%) cases. Furthermore, 8 out of 56 samples (14\%) showed visibly ( $>10$-fold) reduced CD99 expression. Overall, CD99 expression was informative (absent or visibly dimmer than normal) in $84 \%$ of primary plasma cell neoplasm. In the context of minimal residual disease detection, CD99 showed superior utility in separating normal and abnormal plasma cells over currently established antigens CD117, CD81, and CD27 by principal component analysis. Preservation of CD99 expression was strongly associated with cyclin D1 translocation in myeloma $(p<0.05)$. B-cell lymphomas with plasma cell component could be distinguished from myeloma by CD99 expression. In summary, we established that tumor suppressor CD99 is markedly downregulated in multiple myeloma. The loss is highly specific for identification of abnormal cells in primary plasma cell neoplasms, and can be exploited for diagnostic purposes. The role of CD99 in myeloma pathogenesis requires further investigation.
\end{abstract}

Electronic supplementary material The online version of this article (https://doi.org/10.1038/s41379-018-0011-0) contains supplementary material, which is available to authorized users.

\footnotetext{
Ahmet Dogan

dogana@mskcc.org

Mikhail Roshal

roshalm@mskcc.org

1 Hematopathology Service, Memorial Sloan Kettering Cancer Center, New York, NY, USA

2 Department of Epidemiology and Biostatistics, Memorial Sloan
}

\section{Introduction}

CD99(MIC2) is a widely expressed cell surface glycoprotein and functions as a tumor suppressor involved in downregulation of SRC family of tyrosine kinase activity [1-3]. Within the hematopoietic system, CD99 regulates

\section{Kettering Cancer Center, New York, NY, USA}

3 Department of Laboratory Medicine, Memorial Sloan Kettering Cancer Center, New York, NY, USA

4 Department of Pathology, Memorial Sloan Kettering Cancer Center, New York, NY, USA

5 Myeloma Service, Memorial Sloan Kettering Cancer Center, New York, NY, USA 
leukocyte transendothelial migration [4-8], adhesion, and aggregation [9], and facilitates immune surveillance through major histocompatibility complex class I transport from Golgi to cell surface [10].

In hematopoietic lineages, CD99 is most highly expressed on early T and B lymphoblasts, leukemic stem cells, and granulocytic precursors $[3,4,11]$. During B-cell maturation, CD99 expression is lost upon transition from pre-B1 to preB2 stages, and remains low in naive B cells [11-13]. Moderate and high expression of CD99 is seen respectively on tissue memory B cell and plasma cells by immunohistochemical studies [14, 15]. Variable CD99 expression was previously reported in plasma cell neoplasms by immunohistochemistry [16]. Expression of CD99 in low-grade Bcell lymphoma with plasmacytic differentiation has not been investigated.

Flow cytometry immunophenotyping has been established as a powerful diagnostic and monitoring tool in plasma cell neoplasms and non-Hodgkin lymphomas. Compared to immunohistochemical antigen assessment, flow cytometry provides additional diagnostic information including simultaneous assessment of multiple antigens expressed on the same cell, quantitative description of the antigen density, as well as relative proportions of individual populations. Flow cytometry has proven to be more sensitive for evaluation of minimal residual disease and is frequently helpful in distinguishing closely related disease entities [17, 18].

Presence of minimal residual disease post therapy in multiple myeloma by flow cytometry has been consistently associated with inferior outcomes including shorter duration of progression-free survival post treatment [19-26]. The primary goal of the assay is to separate normal residual plasma cells from their neoplastic counterparts. Numerous antigens have been suggested as useful based on differential expression between most normal plasma cells and subsets of their neoplastic plasma cell counterparts. Euroflow consortium has evaluated the utility of numerous antigens by principal component analysis, and identified CD19, CD27, CD38, CD45, CD56, CD81, CD117, CD138, cytoplasmic kappa, and lambda light chains as most useful in this setting [27-29]. This formed the basis for Euroflow two-tube plasma cell minimal residual disease Memorial Sloan Kettering cancer center single-tube 10-color assays [28, 30].

Both low-grade B-cell lymphomas with plasmacytic differentiation and primary plasma cell neoplasms present with neoplastic plasma cell component. While low-grade B-cell lymphomas usually have a B-cell component occasionally, such a definitive B-cell component is not easily demonstrable, or an unrelated B-cell proliferation may be present in the patients with primary plasma cell neoplasm [31]. These cases pose both diagnostic and clinical challenges.

The principal aims of this study were to investigate the clinical utility of CD99 expression (i) in distinguishing normal plasma cells from primary plasma cell neoplasms; (ii) in detection of minimal residual disease in primary plasma cell neoplasms; (iii) in distinguishing plasma cell component of low-grade B-cell lymphomas from primary plasma cell neoplasms.

\section{Methods}

\section{Patient samples}

All specimens were obtained from patients treated or followed at Memorial Sloan Kettering and used in accordance with Memorial Sloan Kettering policies and institutional review board clearance for use of waste samples. For investigation of CD99 expression in B-cell subsets, we analyzed three of each; peripheral blood, bone marrow, and reactive lymphoid tissues. In addition, we evaluated seven normal spleens, four normal tonsils, five reactive lymph nodes by immunohistochemistry. For investigation of CD99 expression on normal and neoplastic plasma cells, we evaluated a total of 10 normal bone marrow aspirates from patients without a history of plasma cell neoplasm, 30 (10 by flow cytometry, 20 by immunohistochemistry) bone marrows involved by B-cell lymphoma with plasmacytic differentiation and 85 bone marrow samples from patients with a history of plasma cell neoplasms, including 56 with the presence of abnormal plasma cells (ranging from 0.0002 to $41.6 \%$ ). Demographic and diagnostic characteristics of the patients are summarized in supplemental table 1 .

\section{Morphologic assessment and immunohistochemistry}

All cases were classified based on clinical characteristics, morphological features, and routine immunophenotyping by immunohistochemistry and flow cytometry. Immunohistochemistry was performed on formalin fixed paraffin embedded sections using monoclonal antibody clone $\mathrm{O} 13$ (Biolegend, San Diego, CA), and standard heat-mediated antigen retrieval/detection on BenchMark ULTRA instrumentation (Ventana Medical Systems, Inc.,Tucson, AZ). Plasma cells were identified based on distribution of CD138 staining and cytologic features. While myeloid forms have been reported to express CD99 by flow cytometry, we observed high intensity staining only in small lymphocytes and plasma cells by immunohistochemistry.

\section{Flow cytometry}

Samples for analysis of CD99 expression on B-cell subsets were stained and analyzed using validated laboratory procedures. Antibody combinations are listed in supplemental 
table 2. Samples were stained with the antibody cocktail for 15 min, lyse-fixed (10\% ammonium chloride lyse $+0.25 \%$ formaldehyde) for $15 \mathrm{~min}$, and washed with PBA solution (phosphate buffered saline $+0.3 \%$ bovine serum albumin $+0.1 \%$ percent sodium azide preparation) prior to acquisition. The samples were acquired on standardized FACS Canto 10-color instruments (BD Biosciences, San Jose, CA), and analyzed using custom Woodlist software (gift of Brent Wood, University of Washington).

For plasma cell analysis, all samples were stained using a standardized procedure as described previously for Memorial Sloan Kettering single-tube 10-color method, and acquired on FACS CANTO 10-color instruments [30, 32]. The abnormal plasma cell population immunophenotype was first identified by Memorial Sloan Kettering single 10-color tube assay using antibody combination described in supplemental table 2. The CD99 was then substituted for kappa FITC/lambda PE light chain antibodies in the investigational panel for evaluation of CD99 (supplemental table 2). The abnormal plasma cells were gated based on known aberrant antigen expression from the current 10-color tube. CD99 expression was not used in gating to avoid bias.

\section{Cytogenetics/FISH}

Fluorescence in situ hybridization (FISH) for $t(11 ; 14)$ (CCND1/IGH) was performed on bone aspirate samples enriched for CD138-positive plasma cells using a Dual color/Dual Fusion probe for CCND1(11q13) /IGH(14q32) (Abbott Molecular), and standard clinical protocols.

\section{Statistical analysis}

Infinicyt $^{\mathrm{TM}}$ Flow Cytometry Data Analysis Software (Cytognos SL, Salamanca, Spain) was used for principal component analysis. Automatic population separator based on principal component analysis [6] was used to determine the relative contribution of CD99 to the first principal component separating normal from abnormal plasma cell clusters in the context of CD138/CD38/CD45/CD56/ CD117/CD19/CD81/CD27. Three database groups were created as follows: normal plasma cell group, abnormal plasma cell group, and residual normal plasma cells in myeloma samples. The normal plasma cell group was created based on 10 normal samples from patients with no history of plasma cell neoplasm. The internal normal plasma cells were gated within the patient samples that had a residual normal population with both normal immunophenotype and an abnormal plasma cell population. For all 56 abnormal samples, we recorded the contribution of variation of each antigen to principal component 1 vector separating abnormal plasma cells from normal plasma cells.

\section{RNA expression analysis}

A total of 813 multiple myeloma samples profiled for translocations and gene expression using whole-genome sequencing and RNA-seq were downloaded from the Multiple Myeloma Research Foundation CoMMpass study [33]. Cytogenetic data was curated based on whole-genome sequencing data using internally developed tools at TGen. Gene level expression data were quantified using Cufflinks and isoform level expressions were estimated using Kallisto and used for downstream analysis. This quantification was based on Ensembl v74 transcript models.

\section{Results}

\section{CD99 is tightly controlled during B-cell maturation, and is upregulated during plasma cell transition}

We first evaluated expression patterns of CD99 in normal bone marrows, blood, and lymphoid tissues by flow cytometry. CD19-positive B cells were subdivided into pre-BI (bone marrow immature $\mathrm{B}$ cells with brightest expression of CD10 and dim CD45), pre-BII (bone marrow immature B cells with intermediate expression of CD10 and CD45), naive $\mathrm{B}$ cells $\left(\mathrm{CD} 45^{\text {bright(br) }}, \mathrm{IgD}+, \mathrm{CD} 27-\right)$, germinal center B cells (lymh node B cells with intermediate expression of CD10 and CD38), memory B cells (CD45 ${ }^{\text {br }}$, $\mathrm{CD} 27+$, IgD-), and plasma cells/plasmablasts (CD38 br/ CD24-). As previously described [11], CD99 expression is rapidly lost upon transition from pre-BI to pre-BII early $\mathrm{B}$ cells in the bone marrow. CD99 expression remains low on naive $\mathrm{B}$ cells in bone marrow, peripheral blood, and lymph nodes. No expression of CD99 was seen in lymph node germinal center B cells. CD99 expression increased on memory B cells in peripheral blood and lymph nodes, and reached the highest levels comparable to those seen in preB1 B cells on plasma cells/plasmablasts in lymph nodes and marrow samples. The findings are summarized in Fig. 1a, b.

To further evaluate the findings in the context of lymphoid tissue architecture, we analyzed spleen (2), tonsil (3), and reactive nodes (5) by immunohistochemistry. In concordance with flow cytometric findings, germinal center B cells showed dim to absent staining, mantle zones (consisting of antigen inexperienced B cells) showed dim staining, and splenic marginal zones showed moderate staining for CD99 (Fig. 1c). Furthermore, CD99 was uniformly expressed in reactive marrow plasma cells by immunohistochemistry (Fig. 1d). 
a
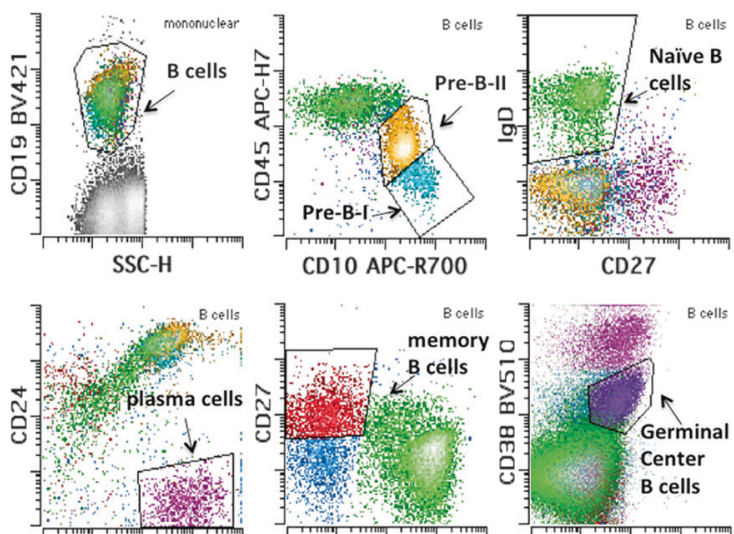

CD38 BV510

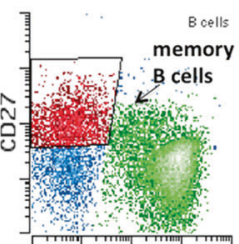

$\lg \mathrm{D}$
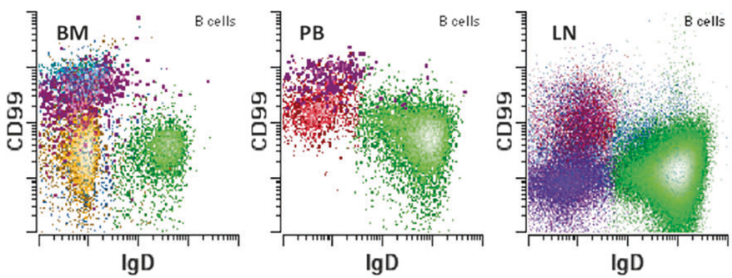

$\lg D$

Fig. 1 a Representative example of CD99 expression through B-cell maturation in bone marrow, blood and lymph node. Early (pre-B-I) hematogones (light blue) show high expression, while late (pre-B-II) (yellow), naive B (green), and germinal center (GC)-B cells (dark purple) show dim to negative expression. The expression is regained on memory B cells (red) and is further increased on plasma cells (purple, emphasized for clarity in selected panels). b Summary of CD99 expression (mean fluorescence intensity) in pre-B-I, pre-B-II,

\section{Normal bone marrow plasma cells express high levels of CD99, while majority of neoplastic plasma cells lose CD99 expression}

We performed further analysis to investigate whether CD99 expression pattern was different between normal and neoplastic plasma cells. We first evaluated 10 bone marrow samples from patients with no history or clinical, morphologic, or laboratory evidence of plasma cell neoplasms. CD99 was brightly and uniformly expressed on normal plasma cells, with no detectable normal plasma cells showing absence of CD99 expression (Fig. 2a). We then analyzed 56 samples from patients with a history of primary plasma cell neoplasm who had abnormal plasma cells in the sample. Abnormal plasma cells from the samples were gated based on the aberrant immunophenotype identified from Memorial Sloan Kettering single-tube 10-color plasma cell assay. Residual normal polytypic plasma cells with apparent normal immunophenotype were gated separately, if present (42 cases). In contrast to the uniform bright expression on normal plasma cells, CD99 expression on neoplastic plasma cells was lost in 39 out of $56(69.6 \%)$ cases. Furthermore, eight out of 56 samples (14\%) showed
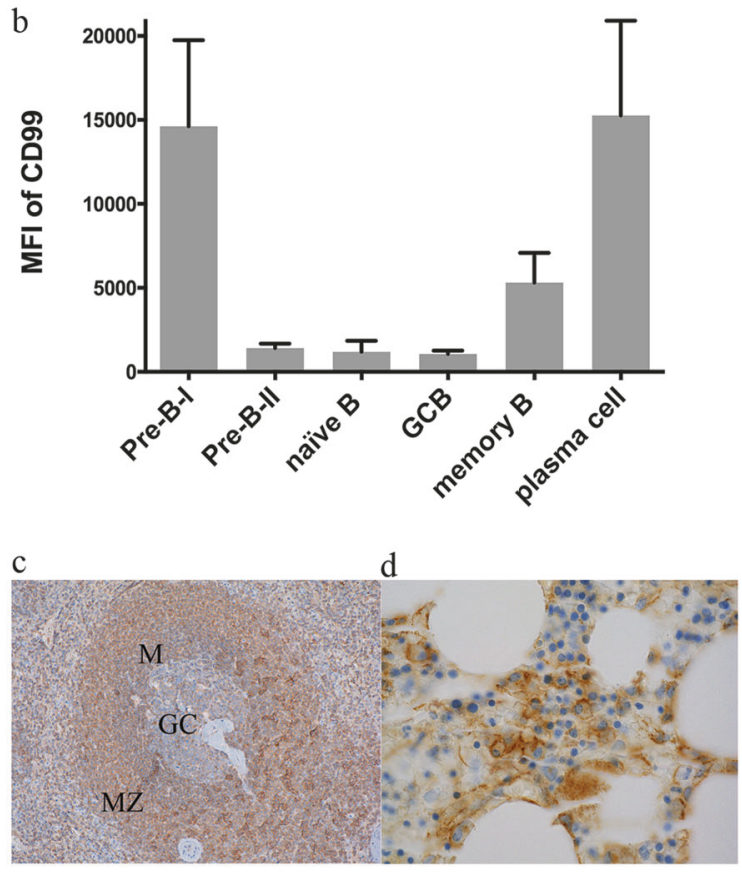

naïve B, GCB, memory B, and plasma cells is shown. c Representative example of CD99 immunohistochemistry staining in normal spleen is shown. Mantle cell (M) composed predominantly of naive B cells and germinal centers show low level staining, while marginal zones (MZ) composed of memory B cells show increased staining $(\times 200)$. d Representative example of CD99 immunohistochemistry staining in reactive polyclonal plasmacytosis. CD99 stains reactive plasma cells $(\times 1000)$

visibly (>10-fold) reduced CD99 expression. Overall, CD99 expression was informative (absent or visibly dimmer than normal) in $84 \%$ of primary plasma cell neoplasm samples (Fig. 2b, c), and showed high specificity and sensitivity for myeloma identification area under the curve for receiver-operator characteristic curve for primary plasma cell neoplasm vs. myeloma was 0.95 (95\% confidence interval 0.91-1, $p<0.05$ ) (Fig. 2d). CD99 expression was slightly, but significantly $(p<0.05)$ increased on residual normal plasma cells in myeloma samples as compared to samples with no plasma cell neoplasms, arguing against therapy-associated loss of CD99 (Fig. 2c).

\section{CD99 shows utility as minimal residual disease antigen for myeloma, and may be useful in identifying abnormal plasma cells by immunohistochemistry}

We further compared the utility of CD99 analysis to wellestablished myeloma minimal residual disease antigens recommended by Euroflow. Principal component analysis was used to calculate percent contribution of each antigen to separation of residual normal plasma cells clusters from 

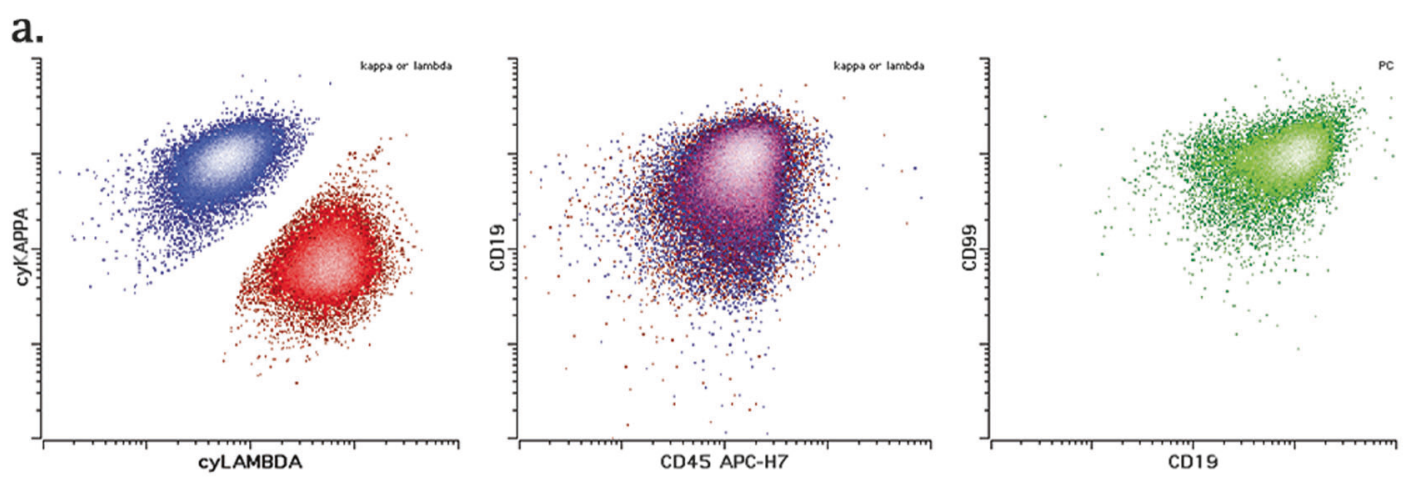

b.
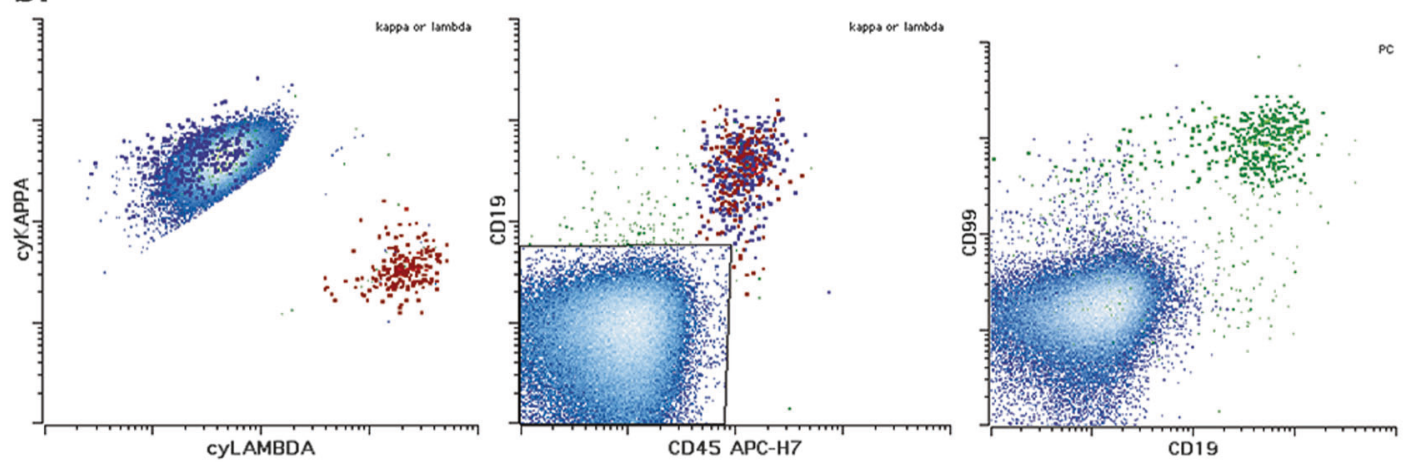

c.

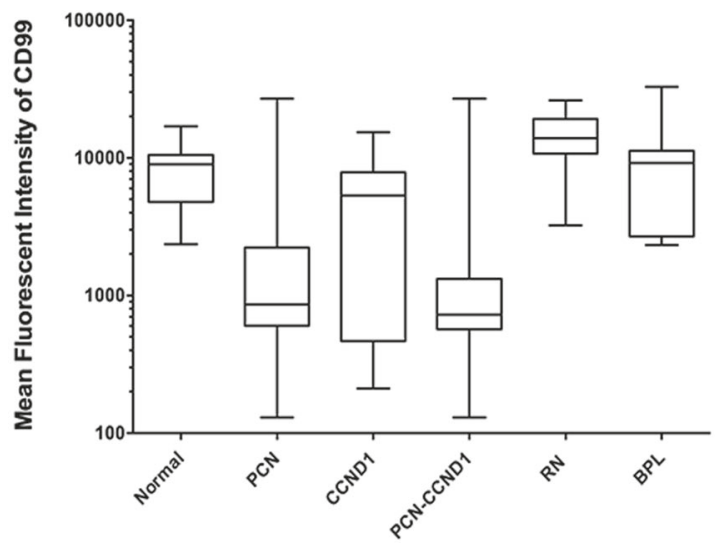

Fig. 2 a Representative example of normal CD99 expression in reactive bone marrow plasma cells is shown. Normal plasma cells gated from tube 1 (kappa expression-blue, lambda expression-red) and from tube 2 (green) show high CD99 expression. b Representative example of neoplastic plasma cells (light blue) show markedly reduced to absent CD99 expression. In contrast, residual normal plasma cells (emphasized) show bright CD99 expression. c Summary of CD99 expression (mean fluorescence intensity) in normal plasma cells,

abnormal plasma cells. To assure that the internal residual normal plasma cell population is appropriate as a normal control, all gated internal normal plasma cells were merged and compared to a group of 10 samples form patients with no history of plasma cell neoplasm. These two groups could not be separated by principal component analysis (Fig. 3a). In contrast, abnormal plasma cells could be fully separated d.

ROC curve: Myeloma vs. Normal CD99

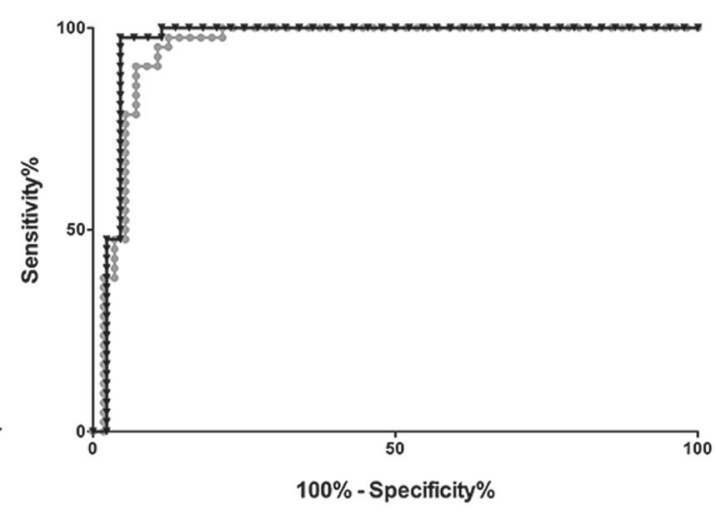

neoplastic (PCN), PCNs with CCND1 translocation (CCND1), without CCND1 translocation (PCN-CCND1), residual normal (RN) plasma cells in samples from patients with PCN, and in B-cell lymphoma with plasmacytic differentiation (BPL) is shown. d Receiver-operator characteristic curve curves for myeloma vs. normal plasma cells are shown for all neoplastic samples (circles) and those without CCND1 translocation (triangles)

from their normal counterparts using principal component one alone (Fig. 3b). Principal component one contributions of each antigen to the separation were recorded (Fig. 3c). Based on the percent contribution to principal component one, CD99 ranked fourth behind well-established antigens CD19, CD45, and CD56 and ahead of CD117, CD27, CD81, and CD38. 


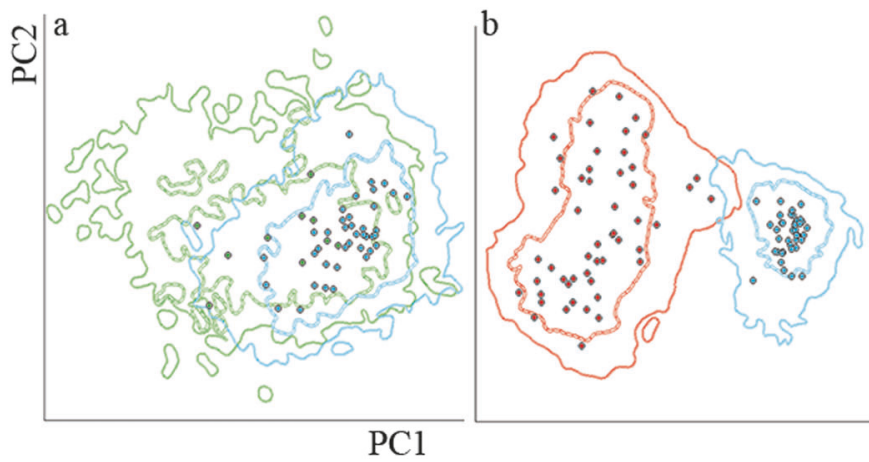

\begin{tabular}{|c|c|}
\hline Markers & \%Contribution to PC1 \\
\hline CD19 & 19.87 \\
\hline CD45 & 13.51 \\
\hline CD56 & 12.36 \\
\hline CD99 & 10.39 \\
\hline CD117 & 10.05 \\
\hline CD27 & 9.02 \\
\hline CD81 & 8.97 \\
\hline CD138 & 4.16 \\
\hline CD38 & 3.48 \\
\hline
\end{tabular}

Fig. 3 a Principal component analysis was performed to compare residual normal plasma cells (blue density plots, means indicated by blue circles) in patients with myeloma and plasma cell from reactive marrows (green). No separation was observed. b Principal component analysis was performed to compare normal plasma cells (blue density

\section{CD99 is retained on majority of CCND1-IGH translocated cases}

In order to evaluate whether CD99 retention is associated with a specific genomic group of myeloma, we compared CD99 status on myeloma cells with the patients' cytogenetic data. Among abnormal myeloma samples that retained CD99 expression, a majority (six out of eight) showed CCND1-IGH fusion/t $(11 ; 14)$. Furthermore, a majority (7 out of 12) samples with CCND1-IGH/t $(11 ; 14)$ expressed CD99, while only ( 2 out of 45$) t(11 ; 14)$-negative cases expressed CD99 ( $p<0.01, \chi^{2}$-test). The mean florescence intensity of CD99 on the abnormal plasma cells was significantly different between CCND1-IGH/t $(11 ; 14)$-positive and -negative cases $(p<0.05)$ (Fig. 2b, Fig. 4a). We further confirmed the finding by analyzing CD99 transcript expression in a large myeloma cohort [33]. The 813 myeloma samples were grouped into $t(11: 14)$ positive and wild type using the cytogenetic data. A total of 176 samples were classified as $t(11: 14)$ positive, whereas 637 were $t(11: 14)$ wild type. As expected significant CCND1 overexpression was seen in the CCND1 translocated group compared to the rest of myeloma samples (Fig. 4b). To determine if there is a significant difference in CD99 gene expression between $t$ (11:14)-positive and -negative cases, a $t$ test was performed. CD99 expression was significantly reduced in $t(11 ; 14)$ negative cases $(p$ value $=2.13 \mathrm{e}-20)($ Fig. $4 \mathrm{c})$.

\section{CD99 is uniformly expressed in plasma cells in the setting of low-grade B-cell lymphoma with plasmacytic differentiation}

Distinction between primary plasma cell neoplasm and lowgrade B-cell lymphoma with plasmacytic differentiation is occasionally challenging, particularly in $t(11 ; 14)$-negative cases where cyclin D1 stain may not be informative. To evaluate if CD99 could be useful in distinguishing between plots, means indicated by blue circles) and neoplastic plasma cells. Full separation could be seen using principal component one component alone. c Relative (percentage) contributions to principal component one separation are listed

primary plasma cell neoplasm and lymphomatous plasma cells, we studied 10 cases of B-cell lymphoma with plasmacytic differentiation by flow cytometry and 30 cases by immunohistochemistry. CD99 was universally retained on the abnormal plasma cells from B-cell lymphoma cases by flow cytometry (Fig. 5a) and immunohistochemistry (Fig. 5b). By flow cytometry, CD99 antigen intensity was significantly higher compared to abnormal plasma cells from myeloma (2731 vs. 9673), $p<0.05$ (Fig. 2b) with area under the curve of $0.88(0.79-0.96, p<0.05)$ (Fig. 5c). The distinction was enhanced once $t(11 ; 14)$ cases were removed (2008 vs. 9673) (Fig. 2b), with a area under the curve of $0.93(0.85-1, p<0.05)$ (Fig. 5c). Thus, CD99 is useful in differentiating primary plasma cell neoplasm from lymphoma with plasma cell component in the bone marrow.

\section{Discussion}

This study has demonstrated that in contrast to normal plasma cells, plasma cells in primary plasma cell neoplasm show marked reduction in CD99 expression. The finding builds upon prior studies indicating variable expression of CD99 in multiple myeloma by immunohistochemistry [16]. Downregulation of CD99 expression appears to be specific for primary plasma cell neoplasms. CD99 was retained on neoplastic plasma cells associated with low-grade B-cell lymphomas and in all normal plasma cells. As the number of low-grade B-cell lymphomas with plasmacytic differentiation, we analyzed by flow cytometry in this study was limited, expanding the cohort could be useful to confirm this finding.

In the context of minimal residual disease analysis, CD99 appears to be at least equivalent to CD27, CD81, and CD117 in distinguishing normal from abnormal plasma cells in primary plasma cell neoplasm by flow cytometry. CD99 may be useful as an additional antigen in the panels 

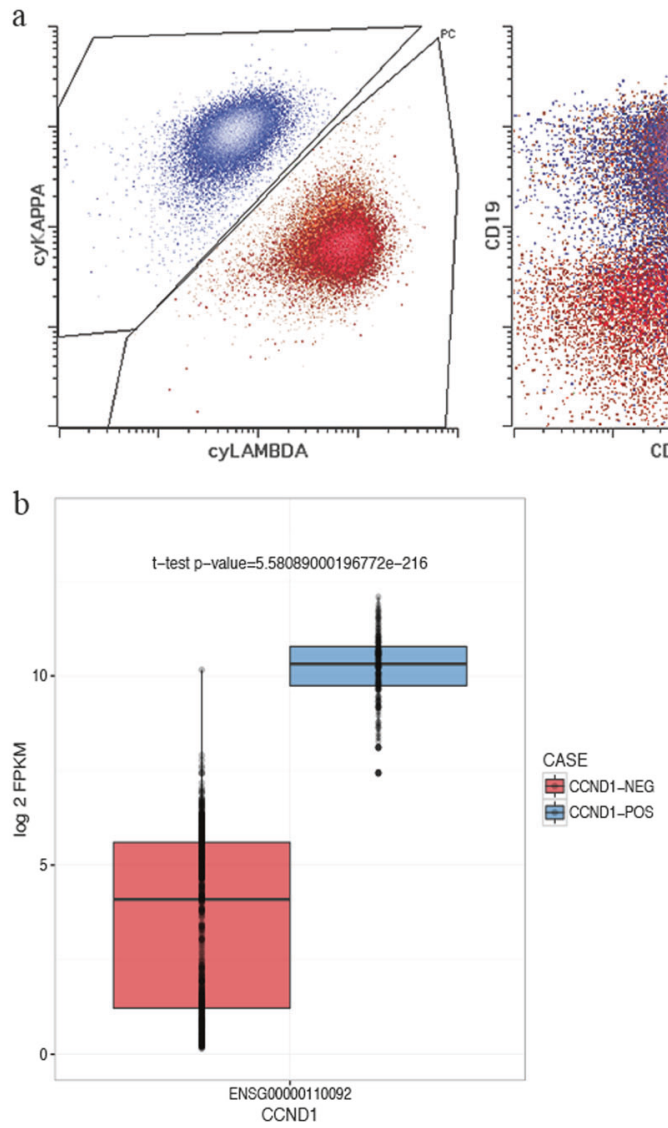

Fig. 4 a Unlike majority of other myeloma cases, those with CCND1 translocation did show frequent loss of CD99 expression in reactive bone marrow plasma cells is shown. Representative example is shown. Lambda-restricted abnormal plasma cells (red) from CCND1 translocated myeloma case showed near normal level of CD99 expression

or as a substitute for one of more antigens used currently. Given the complexity of markers required for successful detection of minimal residual disease, especially in the context antibody therapies that alter expression of plasma cell specific markers such as CD38, availability of a new marker for neoplastic plasma cells may be helpful in designing minimal residual disese assays. Utility of CD99 as a substitute antigen should be evaluated in a larger cohort in a split assay format. In this limited study eliminating CD81 or CD27 in the presence of CD99 did not affect separation between normal and abnormal plasma cells by principal component analysis.

While flow cytometry is clearly the preferred methodology for detection of minimal residual disease, many laboratories may not have access to advanced flow cytometry. In this context, immunohistochemical for CD99 in the context of another stain positively identifying plasma cells such as CD138 could be helpful in identifying neoplastic from normal plasma cells, with absence of CD99 strongly suggestive of primary plasma cell neoplasm.
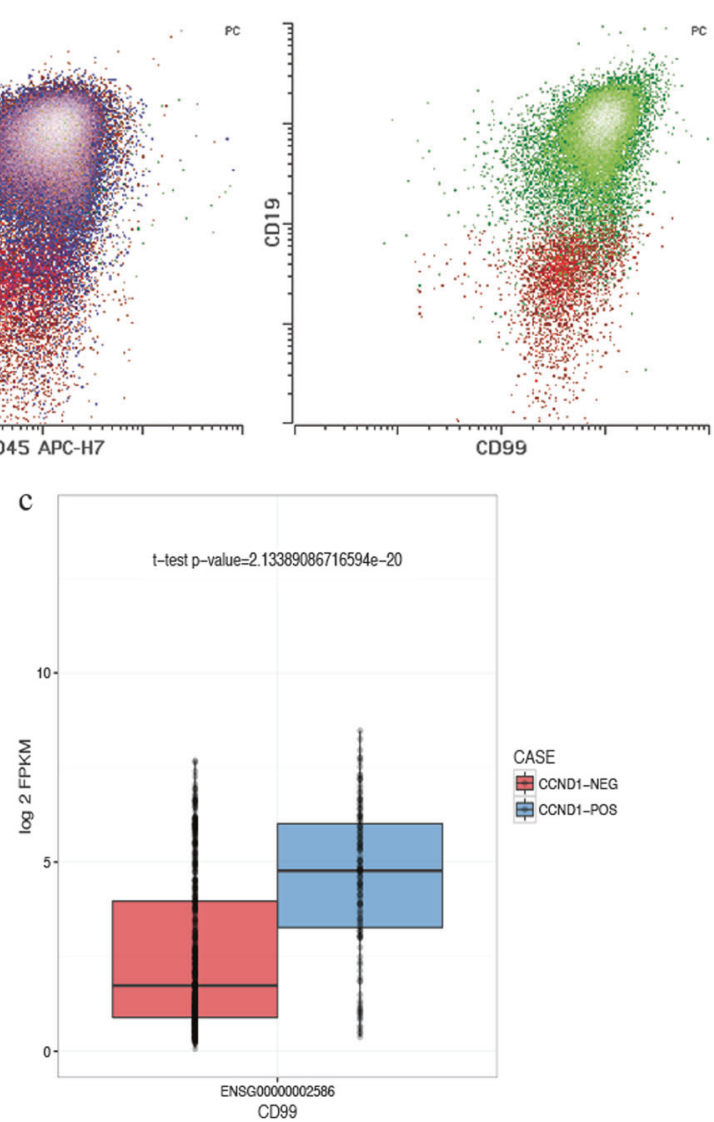

compared to residual normal plasma cells (green). b In an extended cohort of 813 myeloma cases (176 with CCND1 translocation), cyclin D1 was overexpressed in CCND1 translocated cases as expected. c CD99 expression was significantly higher in CCDND1 translocated cases

Multiple myeloma is a genetically heterogeneous disease characterized by frequent chromosomal fusions involving IgH locus and hyperdiploidy [34]. Cases carrying $t(11 ; 14)$ translocation are frequently reminiscent of B-cell lymphoma with plasmacytic differentiation with unique characteristics including frequent expression of B-cell antigen CD20, lymphoplasmacytic morphologic appearance, increased number of circulating plasma cells, occasional expression of IgM heavy chains, and lower levels serum monoclonal immunoglobulin [35]. We now demonstrate that CD99 retention is much more frequent in $t(11 ; 14)$-positive myeloma, suggesting another link between B-cell lymphoma with plasmacytic differentiation and $t(11 ; 14)$-positive myeloma.

\section{Biology}

High frequency of CD99 loss by abnormal plasma cells suggests that CD99 downregulation may be implicated in pathogenesis of multiple myeloma. SRC family of tyrosine 


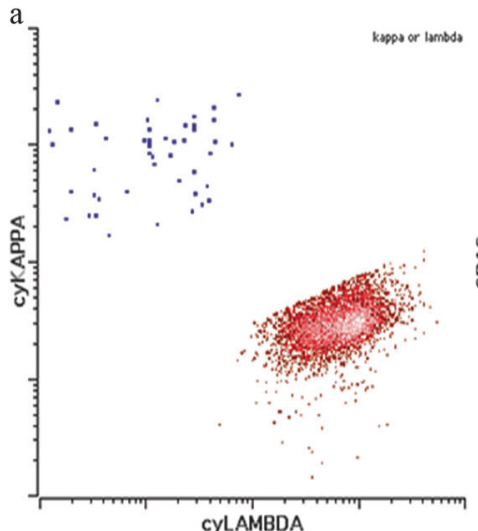

cyLAMBDA

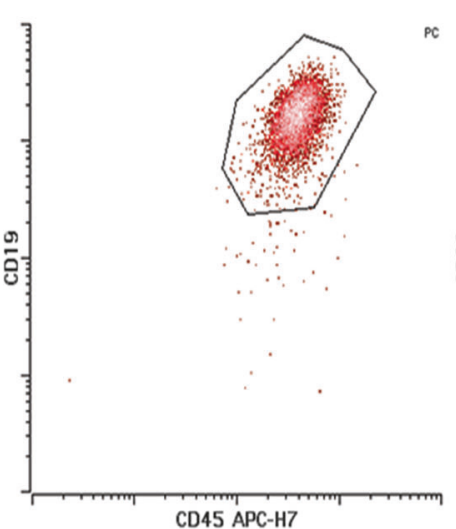

CD45 APC-H7

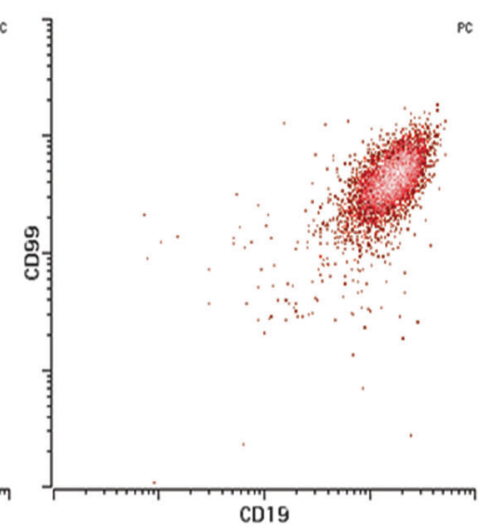

b

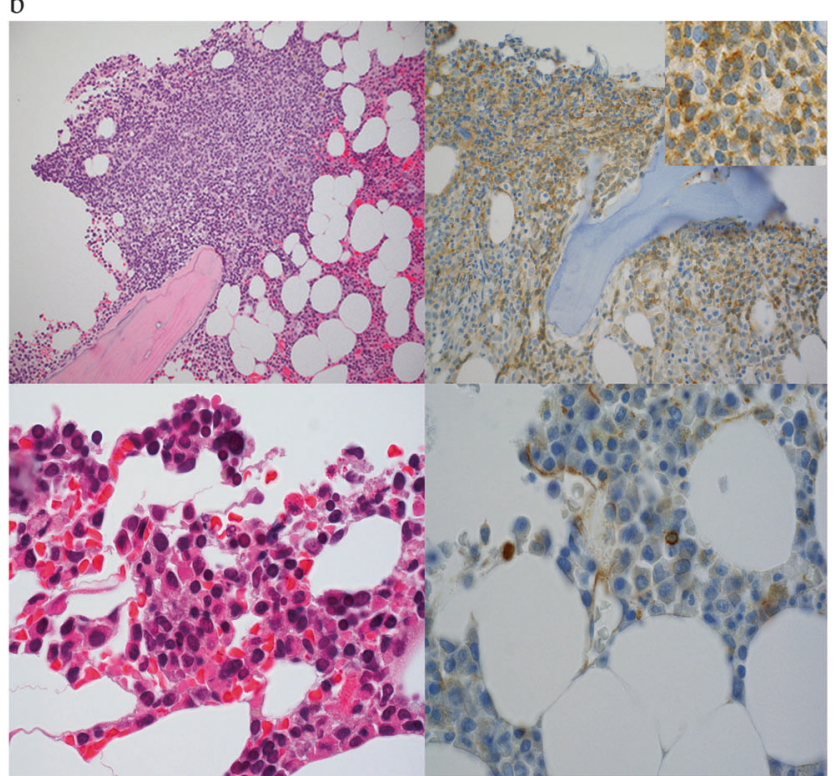

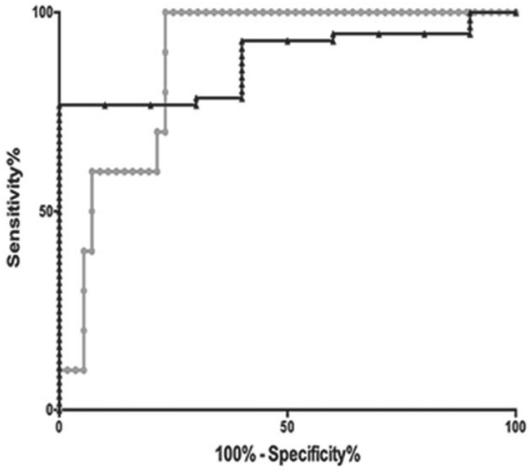

Fig. 5 a Plasma cells in B-cell lymphoma showed bright CD99 expression. Representative example is shown. Lambda-restricted abnormal plasma cells (red) from a patient with LPL showed bright CD99 expression. b CD99 expression could also be detected plasma cells in lymphoma with plasma cell component by immunohistochemistry (top row, $\mathrm{H} \& \mathrm{E} \times 400$ left, $\mathrm{CD} 99 \times 400$ and $\times 1000$ (inset), right). In contrast, CD99 was usually absent in myeloma by immunohistochemistry (bottom row, H\&E $\times 1000$ left, CD99 $\times 1000$, right). c Receiver-operator characteristic curves for myeloma vs. plasma cells from lymphoma are shown for all neoplastic samples (triangles, gray) and those without CCND1 translocation (circles, black) kinase deregulation plays an important part in creating a permissive bone marrow niche for myeloma [36]. CD99 has been shown to downregulate SRC family of tyrosine kinase; this may at least in part be responsible for its purported tumor suppressor activity [2]. Relationship between CD99 loss and SRC family of tyrosine kinase deregulation in myeloma pathogenesis deserves further investigation.

CD99 loss may also modulate migration of plasma cells toward marrow plasma cell niche. Recent study demonstrated a critical role for CD99 in migration toward CXCL12, through ERK-mediated pathway [14]. CD99 expressed on monocytes and neutrophils has also been shown to participate in homophilic interaction with endothelial cells and transendothelial migration [6-8]. Disruption of normal migration pattern across endothelial junction may be important in establishing permissive niche for myeloma growth within the bone marrow. Further investigation is necessary to establish the precise role of CD99 in marrow niche interactions by normal and abnormal plasma cells.

In summary, we established that tumor suppressor CD99 is markedly downregulated in multiple myeloma. The loss is highly specific for identification of abnormal plasma cells in primary plasma cell neoplasms, and can be exploited for diagnostic purposes. The role of CD99 in myeloma pathogenesis requires further investigation. 


\section{Compliance with ethical standards}

Conflict of interest The authors declare that they have no conflict of interest.

\section{References}

1. Zucchini C, Manara MC, Pinca RS, et al. CD99 suppresses osteosarcoma cell migration through inhibition of ROCK2 activity. Oncogene. 2014;33:1912-21.

2. Manara MC, Bernard G, Lollini PL, et al. CD99 acts as an oncosuppressor in osteosarcoma. Mol Biol Cell. 2006; 17:1910-21.

3. Chung SS, Eng WS, Hu W et al. CD99 is a therapeutic target on disease stem cells in myeloid malignancies. Sci. Trans. Med. 2017;9.

4. Imbert AM, Belaaloui G, Bardin F, et al. CD99 expressed on human mobilized peripheral blood CD34+ cells is involved in transendothelial migration. Blood. 2006;108:2578-86.

5. Sullivan DP, Seidman MA, Muller WA. Poliovirus receptor (CD155) regulates a step in transendothelial migration between PECAM and CD99. Am J Pathol. 2013;182:1031-42.

6. Watson RL, Buck J, Levin LR, et al. Endothelial CD99 signals through soluble adenylyl cyclase and PKA to regulate leukocyte transendothelial migration. J Exp Med. 2015;212:1021-41.

7. Lou O, Alcaide P, Luscinskas FW, Muller WA. CD99 is a key mediator of the transendothelial migration of neutrophils. J Immunol. 2007;178:1136-43.

8. Schenkel AR, Mamdouh Z, Chen X, Liebman RM, Muller WA. CD99 plays a major role in the migration of monocytes through endothelial junctions. Nat Immunol. 2002;3:143-50.

9. Hahn JH, Kim MK, Choi EY, et al. CD99 (MIC2) regulates the LFA-1/ICAM-1-mediated adhesion of lymphocytes, and its gene encodes both positive and negative regulators of cellular adhesion. J Immunol. 1997;159:2250-8.

10. Sohn HW, Shin YK, Lee IS, et al. CD99 regulates the transport of MHC class I molecules from the golgi complex to the cell surface. J Immunol. 2001;166:787-94.

11. Dworzak MN, Fritsch G, Buchinger P, et al. Flow cytometric assessment of human MIC2 expression in bone marrow, thymus, and peripheral blood. Blood. 1994;83:415-25.

12. Dworzak MN, Fritsch G, Fleischer C, et al. Comparative phenotype mapping of normal vs. malignant pediatric B-lymphopoiesis unveils leukemia-associated aberrations. Exp Hematol. 1998;26:305-13.

13. Dworzak MN, Fritsch G, Fleischer C, et al. CD99 (MIC2) expression in paediatric B-lineage leukaemia/lymphoma reflects maturation-associated patterns of normal B-lymphopoiesis. Br J Haematol. 1999;105:690-5.

14. Gil M, Pak HK, Lee AN, et al. CD99 regulates CXCL12-induced chemotaxis of human plasma cells. Immunol Lett. 2015;168:329-36.

15. Park CK, Shin YK, Kim TJ, Park SH, Ahn GH. High CD99 expression in memory $\mathrm{T}$ and $\mathrm{B}$ cells in reactive lymph nodes. $\mathrm{J}$ Korean Med Sci. 1999;14:600-6.

16. Shin SJ, Lee H, Jung G, et al. Expression of CD99 in multiple myeloma: a clinicopathologic and immunohistochemical study of 170 cases. Korean J Pathol. 2014;48:209-16.

17. Kumar S, Paiva B, Anderson KC, et al. International Myeloma Working Group consensus criteria for response and minimal residual disease assessment in multiple myeloma. Lancet Oncol. 2016;17:e328-e46.

18. Mailankody S, Korde N, Lesokhin AM, et al. Minimal residual disease in multiple myeloma: bringing the bench to the bedside. Nat Rev Clin Oncol. 2015;12:286-95.
19. Chanan-Khan AA, Giralt S. Importance of achieving a complete response in multiple myeloma, and the impact of novel agents. $\mathrm{J}$ Clin Oncol. 2010;28:2612-24.

20. de Tute RM, Rawstron AC, Gregory WM, et al. Minimal residual disease following autologous stem cell transplant in myeloma: impact on outcome is independent of induction regimen. Haematologica. 2016;101:e69-e71.

21. Landgren O, Devlin S, Boulad M, Mailankody S. Role of MRD status in relation to clinical outcomes in newly diagnosed multiple myeloma patients: a meta-analysis. Bone Marrow Transplant. 2016;51:1565-8.

22. Landgren O, Gormley N, Turley D, et al. Flow cytometry detection of minimal residual disease in multiple myeloma: lessons learned at FDA-NCI roundtable symposium. Am J Hematol. 2014;89:1159-60.

23. Martinez-Lopez J, Lahuerta JJ, Pepin F, et al. Prognostic value of deep sequencing method for minimal residual disease detection in multiple myeloma. Blood. 2014;123:3073-9.

24. Paiva B, van Dongen JJ, Orfao A. New criteria for response assessment: role of minimal residual disease in multiple myeloma. Blood. 2015;125:3059-68.

25. Rawstron AC, Child JA, de Tute RM, et al. Minimal residual disease assessed by multiparameter flow cytometry in multiple myeloma: impact on outcome in the Medical Research Council Myeloma IX Study. J Clin Oncol. 2013;31:2540-7.

26. Rawstron AC, Gregory WM, de Tute RM, et al. Minimal residual disease in myeloma by flow cytometry: independent prediction of survival benefit per log reduction. Blood. 2015;125:1932-5.

27. Flores-Montero J, de Tute R, Paiva B, et al. Immunophenotype of normal vs. myeloma plasma cells: toward antibody panel specifications for MRD detection in multiple myeloma. Cytometry B Clin Cytom. 2016;90:61-72.

28. Flores-Montero J, Sanoja-Flores L, Paiva B et al. Next generation flow (NGF) for highly sensitive and standardized detection of minimal residual disease in multiple myeloma. Leukemia. 2017;31:2094-2103.

29. Rawstron AC, Paiva B, Stetler-Stevenson M. Assessment of minimal residual disease in myeloma and the need for a consensus approach. Cytometry B Clin Cytom. 2016;90:21-5.

30. Royston DJ, Gao Q, Nguyen N, et al. Single-tube 10-fluorochrome analysis for efficient flow cytometric evaluation of minimal residual disease in plasma cell myeloma. Am J Pathol. 2016;14:41-9.

31. Rosado FG, Morice WG, He R, et al. Immunophenotypic features by multiparameter flow cytometry can help distinguish low grade B-cell lymphomas with plasmacytic differentiation from plasma cell proliferative disorders with an unrelated clonal B-cell process. Br J Haematol. 2015;169:368-76.

32. Roshal M, Flores-Montero JA, Gao Q, et al. MRD detection in multiple myeloma: comparison between MSKCC 10-color singletube and EuroFlow 8-color 2-tube methods. Blood Adv. 2017;1:728-32.

33. Lonial S, Yellapantula VD, Liang W, et al. Interim analysis of the Mmrf commpass trial: identification of novel rearrangements potentially associated with disease initiation and progression. Blood. 2014;124:722.

34. Szalat R, Munshi NC. Genomic heterogeneity in multiple myeloma. Curr Opin Genet Dev. 2015;30:56-65.

35. Fonseca R, Blood EA, Oken MM, et al. Myeloma and the $t(11 ; 14)$ (q13; q32); evidence for a biologically defined unique subset of patients. Blood. 2002;99:3735-41.

36. Coluccia AM, Cirulli T, Neri P, et al. Validation of PDGFRbeta and $\mathrm{c}-\mathrm{Src}$ tyrosine kinases as tumor/vessel targets in patients with multiple myeloma: preclinical efficacy of the novel, orally available inhibitor dasatinib. Blood. 2008;112:1346-56. 